\title{
VIII ENCUENTRO DE INVESTIGACIÓN ODONTOLOGÍA UC
}

\section{Escuela de Odontología}

Facultad de Medicina

Pontificia Universidad Católica de Chile

10 de enero, 2020
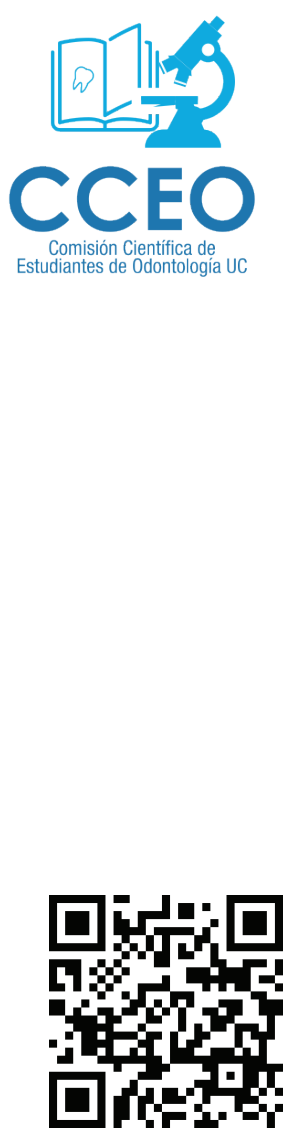
El viernes 10 de enero se realizó en las dependencias de la Escuela de Odontología de la Pontificia Universidad de Chile, el VIII Encuentro de Investigación de Odontología UC. Este evento fue organizado por la Comisión de Investigación de la Escuela, junto a la Comisión Científica de Estudiantes (CCEO UC). En este encuentro, fueron socializados 6 trabajos de investigación en la modalidad de presentación oral y 28 posters, en los que participaron estudiantes de pregrado de nuestra Escuela y de varias Facultades de Odontología de nuestro país. 


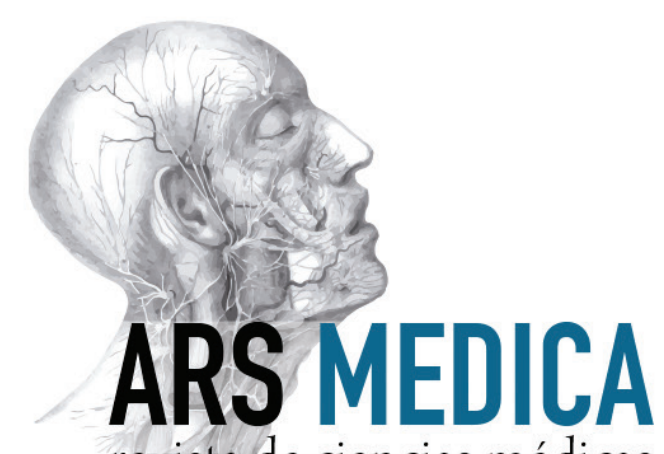
revista de ciencias médicas

COMISIÓN DE INVESTIGACIÓN

ESCUELA DE ODONTOLOGÍA

Dra. Constanza Martínez Cardozo (Directora)

Dra. Bruna Benso

Dr. Sebastián Aguayo

Dr. Humberto González

Dr. Raúl Palacio

Dra. Maite Souyet

Dr. Eduardo Magallón

COMISIÓN CIENTÍFICA DE
STUDIANTES DE ODONTOLOGÍA

Nicolás Santander Ramírez

María Francisca Cortés San Juan

Ivana Vásquez Balazs

Francisca Figueroa Pimentel

Josefina Cid Cid 


\section{CONFERENCIA: LA NATURALEZA DE LA REGENERACIÓN DENTARIA EN VERTEBRADOS}

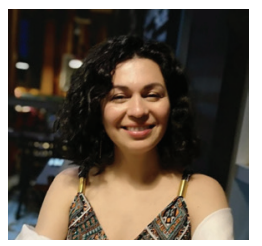

\section{DRA. MARCIA GAETE}

Profesor Asociado

Departamento de Anatomía,

Facultad de Medicina, Pontificia Universidad

Católica de Chile, Santiago, Chile

La naturaleza nos ofrece una variedad de información que puede ser observada para ampliar nuestro conocimiento y aplicarlo en preguntas básicas, tales como la de cómo podemos inducir la regeneración dentaria. Es así como en esta ocasión revisamos los procesos de regeneración dentaria homeostática en vertebrados.

La mayoría de los vertebrados presentan una renovación constante de sus dientes, en donde muchas generaciones de dientes están siendo formadas constantemente. Estos animales se denominan polifiodontes. Otros vertebrados poseen un número limitado de dientes y pueden presentar dos sets dentarios durante su vida, denominándose difiodontes, o un set, denominándose monofiodontes. Surge la pregunta acerca de cuál es la diferencia entre estas diferentes clases de animales, y si de alguna manera podemos convertir un monofiodonte o difiodonte en un polifiodonte o viceversa.

Hipotetizamos que la capacidad constante de regeneración dentaria radica en la mantención de un grupo de células madre dentarias que se ubican en una lámina dental compartimentalizada. Se analiza este sistema en el gecko, cocodrilo, tiburón y serpiente, en los que parte de la lámina dental o una lámina dental completa se asocia a la presencia de células madre, células de ciclo lento que retienen la marca de incorporación de nucleótidos, y la mantención de un subcompartimiento de la lámina con actividad elevada de la vía Wnt beta catenina, relacionada con la mantención de su actividad proliferativa y su permanencia en el tiempo.

En difiodontes, tales como el ser humano y el hurón, se analiza una congruencia entre una lámina dentaria continua portadora de células madre y la activación vía Wnt beta catenina con el proceso de reemplazo dentario. La activación tardía de restos de epitelio odontogénico a nivel gingival se asocia con tumores odontogénicos. La regulación del número dentario puede ser también analizada por métodos de cultivo de dientes en formación, sobre todo en modelos animales no tradicionales. Es así como en serpientes la activación de la vía Wnt aumenta el número de dientes producido en un tiempo determinado, y en el modelo ratón se puede activar una formación de dientes extra cortando la conexión entre los dientes en formación. Esto interrumpe la conexión de la lámina dentaria, reorganiza la reacción-difusión y aumenta el número dentario.
Se concluye que la presencia de lámina dental y la regulación de células madre residentes en ella se asocia a las diferentes presentaciones de de fiodontía.

Por último, se asocia el constante remplazo dentario constante con vertebrados no mamíferos tales como peces, anfibios y reptiles, mientras que los mamíferos tienden a tener un reemplazo limitado de dientes. Hay excepciones a la regla y un mamífero que ha despertado interés por su regeneración dentaria constante es el elefante. En él se forman constantemente dientes en la región retromolar y ellos migran hacia mesial.

En los últimos años se ha ampliado el espectro de animales que se estudian para entender la regeneración dentaria y cada vez más la naturaleza parece aportarnos respuestas a interrogantes básicas para la terapia de la regeneración dental.

\section{CONFERENCIA: ENS 2016-2017: DESAFÍOS EN INVESTIGACIÓN Y VIGILANCIA EPIDEMIOLÓGICA PARA ODONTOLOGÍA.}

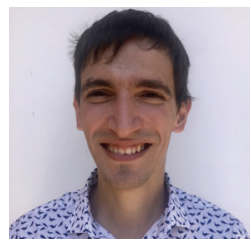

\section{DR. DUNIEL ORTUÑO}

Profesor Docente Asistente Escuela de Odontología, Pontificia Universidad Católica de Chile, Santiago, Chile

La Encuesta Nacional de Salud (ENS) es un instrumento de vigilancia epidemiológica y un insumo indispensable para generación, monitoreo y evaluación de políticas públicas. La ENS 2016-2017 es la tercera versión de este estudio en Chile, donde se abordaron 72 problemas de salud y sus determinantes, incluyendo la salud oral y factores de riesgo asociados. De acuerdo con el modelo de encuestas poblacionales sugerido por la Organización Mundial de la Salud (STEPwise approach), la salud bucal en la ENS 2016-2017 se evaluó a través de dos pasos (steps): 1-cuestionario sobre salud oral (F1) y 2-examen de salud bucal (F2). En este último se abordó el uso de prótesis removibles, el número de dientes en cada maxilar, la presencia de lesiones cavitadas de caries y la pérdida de dientes anteriores. La ENS 2016-2017 mostró una reducción de un 11,7\% en la prevalencia de caries cavitadas en adultos con respecto a los resultados de la ENS 2003, sin embargo, la prevalencia global de esta condición crónica en el país sigue siendo muy elevada (54,6\%). Un importante problema de salud es la pérdida de dientes, situación que aumenta con la edad y es mayor en mujeres, en personas con nivel educacional bajo y en habitantes de zonas rurales del país. En estudios relacionados mostramos que las personas con un nivel educacional alto (12 o más años de estudios), tienen 
13,33 veces más chance de presentar al menos veinte dientes en boca (dentición funcional), comparado con aquellos individuos de nivel educacional bajo. Estos resultados son concordantes con análisis previos como comparaciones multinacionales, que han evidenciado la persistencia de inequidades en salud bucal en Chile. Por otro lado, en la Escuela de Odontología UC, realizamos uno de los subestudios incluidos en el marco de la ENS 2016-2017, concluyendo que a nivel poblacional es factible implementar el autorreporte como un método válido y similar al examen clínico para evaluar el número de dientes en adultos. Estos últimos hallazgos son concordantes con estudios realizados en Japón, Finlandia, Canadá y Estados Unidos, en los cuales se encontraron correlaciones altas y significativas, ajustando por edad y sexo. En la ENS 2016-2017 por primera vez se determinó la pérdida de dientes anteriores, considerando el impacto que tiene esta situación en la calidad de vida de las personas y la necesidad de generar programas que aborden soluciones. Un $29,4 \%$ de la población presentó falta de dientes anteriores en el maxilar superior y un 18,5\% en el maxilar inferior. Nuevamente existen diferencias por grupos, siendo más probable la pérdida de dientes anteriores en mujeres, en individuos de nivel educacional bajo y en habitantes de zonas rurales. Estudios preliminares nuestros indican que la pérdida de dientes se asocia a la calidad de vida de las personas, especialmente en los ámbitos de actividades diarias, autocuidado y movilidad. En conclusiones, la ENS es un instrumento para considerar con mayor énfasis en las políticas públicas dirigidas a abordar la salud bucal, especialmente en la formulación de los Objetivos Sanitarios de la nueva década. 


\section{Trabajos presentados en moralidad oral}




\section{Efecto antimicrobiano de nanopartículas a base de polisacáridos/timol sobre patógenos de la cavidad oral}

\author{
Alan Polanco ${ }^{1}$, Nicol Flores ${ }^{1}$, Marlen Barreto ${ }^{2}$, Nelson Caro ${ }^{3}$ \\ 'Carrera de Odontología, Universidad Autónoma de Chile. Santiago, Chile. \\ ${ }^{2}$ Instituto de Investigaciones Biomédicas, Universidad Autónoma de Chile. \\ Santiago, Chile. ${ }^{3}$ Centro de Investigación Austral Biotech, Universidad Santo \\ Tomás. Santiago, Chile.
}

Introducción: Muchas patologías de la cavidad oral son originadas por patógenos con capacidades defensivas bastante elevadas debido a sus características microbiológicas, lo que dificulta en gran medida su erradicación. De los microorganismos multiresistentes presentes en la cavidad oral, enterococcus faecalis y cándida albicans son los más prevalentes, y debido a la dificultad en su erradicación, el tratante debe manejar alternativas terapéuticas en caso de fracasar la terapia de erradicación convencional. Por otra parte, la aplicación de nanopartículas ha mostrado resultados bastante interesantes en el control de procesos infecciosos, sin embargo, su utilización en la cavidad oral carece de referencias.

Objetivo: Evaluar la actividad antimicrobiana de nanopartículas a base de polisacáridos de quitosano y betaglucano con moléculas bioactivas de timol encapsulado sobre enterococcus $s p$ y cándida albicans.

Metodología: Seaplicó diferentes formulaciones de nanopartículas sobre cultivos (estado planctónico y biopelículas) de enterococcus sp y cándida albicans (previamente caracterizadas mediante procesos bioquímicos) yse analizó su efecto mediante absorbancia de densidad óptica a 600nm en el sistema tecam infinite pro M200. La cantidad de nanopartículas añadida se determinó mediante pruebas de CMI. Las muestras se dividieron en 7 grupos de estudio: (1) solución salina (PBS) (control negativo). (2) clorhexidina al 2\% (control positivo). (3) nanopartículas de quitosano (NQo). (4) nanopartículas hibridas de quitosano/betaglucano con timol (NBQT) (5) nanopartículas de betaglucano con timol (NBG-T). (6) nanopartículas de quitosano con timol (NQT). (7) nanopartículas de betaglucano (BG). Grupos 3,4,5,6 y 7 corresponden al grupo experimental. Las nanopartículas utilizadas fueron fabricadas y caracterizadas en los laboratorios australbiotech de la universidad santo tomas.

Resultados: La densidad óptica del grupo experimental y control positivo respecto al control negativo fue significativamente menor $(p<0.05)$ tanto para formas planctónicas como biopeliculas de enterococcus sp y cándida albicans.

Conclusiones: Nanopartículas a base de polisacáridos con y sin timol encapsulado, son capaces de inhibir el crecimiento tanto para enterococcus sp como para cándida albicans in vitro.

\section{Prevalencia de enfermedad periodontal y autopercepción de salud en adultos mayores hospitalizados en San Bernardo}

\author{
Maximiliano Sáez', Gloria Maurer', Matías Hagn' 'Daniela Olguín', \\ Claudia Cristoffanini ${ }^{1}$ \\ ${ }^{1}$ Facultad de Odontología, Universidad de los Andes. Santiago, Chile.
}

Introducción: En Chile viven más de 2 millones de adultos mayores (mayor o igual a 60 años) y para el 2025 se proyecta un aumento de un 50\%. La periodontitis es aquella enfermedad inflamatoria multifactorial que provoca pérdida dentaria y está presente en un 69\% de adultos mayores entre los 65-74 años. Actualmente no existen estudios de prevalencia de periodontitis en adultos mayores hospitalizados, ni de valoración del programa GES 60 años, siendo estos factores indispensables para el inicio de un enfoque preventivo/terapéutico.

Pregunta de investigación: ¿Cuál es la prevalencia de enfermedad periodontal y la autopercepción de salud oral en los adultos mayores de 60 años, hospitalizados en el servicio de Medicina Interna (MI) del Hospital Parroquial de San Bernardo (HPSB), Santiago de Chile?

Materiales y métodos: Se realizó un estudio de corte transversal, donde se seleccionaron 153 voluntarios aleatorios hospitalizados en el servicio de MI del HPSB durante marzo a septiembre del año 2019, mediante una encuesta que considera variables sociodemográficas, médico-odontológicas, clínicas, periodontales y cualitativas de autopercepción, que posteriormente se analizaron mediante Excel.

Resultados: La mayoría de los participantes eran hombres, de nivel socioeconómico y nivel educacional bajo. 100\% de los participantes presentó enfermedad periodontal (84,3\% periodontitis y $15,6 \%$ gingivitis). A la mayoría de los participantes le preocupa su salud oral, creen que su salud oral es deficiente y asocian esta situación a la poca educación sobre salud que han recibido a lo largo de su vida. $69,2 \%$ de los participantes no conoce el beneficio GES 60 años.

Conclusiones: Se requiere informar más a la población sobre el programa GES y la realización de nuevos estudios de prevalencia en patologías orales de pacientes hospitalizados, para así estudiar la necesidad de un equipo odontológico en servicios de $\mathrm{MI}$ que ayude en el diagnóstico y prevención de éstas. 


\section{Evaluación de impacto del Programa Más Sonrisas para Chile 2016-2017 mediante el cuestionario OHIP7}

Francisco Moreno ${ }^{1}$, Claudio Carrasco ${ }^{1}$, Juan Pablo Vargas ${ }^{1}$, Duniel Ortuño Borroto', Sebastián Prado², Alexis Ahumada²

'Escuela de Odontología, Facultad de Medicina, Pontificia Universidad Católica de Chile. Santiago, Chile. ${ }^{2}$ Dirección de Salud, Ilustre Municipalidad de Recoleta. Recoleta, Chile.

En Chile, desde el año 2015 existe el programa Más Sonrisas Para Chile (MSPCH), el cual tiene como propósito entregar atención odontológica a mujeres de 20 o más años, inscritas en FONASA. El impacto del programa se evalúa mediante la encuesta de calidad de vida OHIP7, la cual consiste en 7 preguntas de autorreporte, cada una representando una dimensión diferente con respecto a la calidad de vida. Las respuestas son ordinales, entre 0 y 4 , donde 0 = nunca, $1=$ casi nunca, $2=$ ocasionalmente, $3=$ casi siempre y $4=$ siempre. Así, a menor puntaje, mayor calidad de vida.

El objetivo de este estudio fue evaluar el impacto del programa MSPCH en sus beneficiarias comparando sus puntajes OHIP7 prepost intervención.

Se obtuvieron las bases de datos MSPCH 2016 y 2017 del país, a través del DEIS, las cuales contenían las respuestas pre y post tratamiento y los puntajes OHIP7, ( $\mathrm{N}=29.253,154$ casos perdidos). Los análisis estadísticos se realizaron mediante el programa SPSS, ajustando los datos por año, edad y macrozona.

La tasa de consistencia interna de la encuesta fue alta (alfa de Cronbach $=0,879$ ). Se observó un impacto positivo del programa MSPCH, las personas que mantuvieron o disminuyeron su puntaje OHIP7, fue superior a un $90 \%$ en ambos años. En las 7 dimensiones de la encuesta OHIP7 se encontró que los puntajes disminuyeron de manera significativa (Test de Wilcoxon, $p<0,001)$. En grupos etarios el mayor impacto se observó en mujeres de 20 a 24 años (94\% de impacto, $p<0,001$ ). Según macrozona, la Región Metropolitana fue la única que no tuvo un impacto por sobre el $90 \%$.

Se concluyó que, en Chile, la calidad de vida de las mujeres, medida a través de la encuesta OHIP 7, mejora significativamente después de las intervenciones realizadas en el programa MSPCH.

\section{La atención primaria desde los determinantes sociales en primera infancia: un desafío pendiente}

Daniela Guzmán', Carla Madrid', Paulina Humeres

'Escuela de Odontología, Facultad de Medicina, Pontificia Universidad Católica de Chile. Santiago, Chile.

Introducción: En Chile el daño por caries en los niños preescolares demuestra la inequidad social en salud. A los 2 años, la prevalencia de caries en niños de familias de nivel socioeconómico alto es de $9,5 \%$ y $20,3 \%$ en niños de familias vulnerables. El cuidado en salud oral recae fundamentalmente en las madres en los primeros años de vida, donde sus conocimientos y creencias contribuyen en la adquisición de hábitos. Si bien se han implementado modelos enfocados en prevención e integralidad en la atención, la evidencia señala que es importante que el tratante conozca los determinantes sociales del paciente, para comprender la enfermedad desde otras dimensiones y lograr un cambio significativo en salud. ¿Cuáles son algunos de estos determinantes presentes en la vida de los niños preescolares que se atienden en la Atención Primaria?

Métodos: Se realizó una encuesta estructurada y validada a 65 cuidadores de niños entre 2 a 5 años, usuarios de CESFAM de Puente Alto, se analizó de manera cuantitativa y descriptiva a través del programa SPSS V24.0

Resultados: $49 \%$ madres son dueñas de casa, $44.6 \%$ tienen enseñanza media completa. $70,8 \%$ cree que las caries se previenen con cepillado y visitas al dentista. Sin embargo, el $25 \%$ de los niños se acostó la noche anterior a la encuesta sin cepillarse los dientes y un $32.3 \%$ no ha asistido al dentista. $29.3 \%$ de los cuidadores considera difícil el momento del cepillado de su hijo y $23.8 \%$ termina obligando al hijo para que lo realice.

Conclusión: Si bien existe conocimiento entre las madres sobre medidas de cuidado oral, la información por si sola no garantiza el adecuado desarrollo de hábitos. Los determinantes sociales ayudan a comprender la realidad del paciente y a planificar estrategias individualizadas que apunten a mejorar su salud.

Financiamiento: Concurso Interno de investigación en Odontología 2017 


\section{Severidad e índice significante de caries en niños beneficiarios del programa sembrando sonrisas de La Florida}

José Antonio Plaza', Javiera Olivares', Javiera Rodríguez'1, Diego Angulo ${ }^{2}$

${ }^{1}$ Facultad de Odontología, Universidad de Chile. Santiago, Chile. ${ }^{2}$ Departamento de Salud, Corporación Municipial de la Florida. Santiago, Chile.

Introducción: En el marco del modelo de atención integral de salud familiar y comunitario nace el programa Sembrando Sonrisas (SS), cuyos objetivos son mantener y mejorar la salud oral de la población parvularia, a través de la promoción de hábitos saludables, y la aplicación de medidas de protección, además del trabajo intersectorial con la comunidad.

Hipótesis: La población pre-escolar beneficiaria durante el 2018 del programa Sembrando Sonrisas de la Florida presenta menor ceo-d, COP-D e índice significante de caries que la población nacional.

Metodología: Este estudio descriptivo de corte transversal comprende el universo de párvulos pertenecientes a establecimientos de la Junta Nacional de Jardines Infantiles (JUNJ), Fundación Integra y niveles de pre-kínder y kínder de escuelas municipalizadas y subvencionadas (MINEDUC) de La Florida. Se recolectaron datos del programa SS para estudiar severidad de caries. Se traspasaron los datos a una planilla Excel y se procesó en el programa Stata 15.0 utilizando un modelo de regresión logística.

Resultados: La muestra de estudio corresponde a 3.164 pre-escolares, de los cuales el $49,53 \%$ son mujeres y el $50,47 \%$ hombres, con un promedio de edad de 3,5 años. El promedio del ceo-d fue de 1,37 y el COP-D fue de 0,01 encontrándose correlación positiva entre el aumento en edad con un incremento en la severidad de caries. El índice significante de caries fue de 4,02.

Conclusión: Los pre-escolares de la comuna de la Florida tienen una severidad de caries menor a la nacional. Sin embargo, a pesar del bajo valor general de la muestra, existe un grupo más afectado, reflejado por el índice significante, que presenta un índice ceo-d sumamente alto, cercano al doble de los datos nacionales publicados por el MINSAL.

\section{Caracterización y síntesis de derivados de productos de origen natural con potencial actividad antibacteriana}

Francisca Arcos ${ }^{1}$, Miguel Zágarra² ${ }^{2}$ Sebastián Aguayo ${ }^{1}$, Bruna Benso․

'Escuela de Odontología, Facultad de Medicina, Pontificia Universidad Católica de Chile. Santiago, Chile. 'Escuela de Odontología, Facultad de Medicina, Pontificia Universidad Católica de Chile. Santiago, Chile.

La resistencia antimicrobiana es un problema de salud pública mundial, es por este motivo que sintetizamos un conjunto de nuevas chalconas, con el objetivo de identificar posibles efectos antibacterianos contra Streptococcus mutans UA159. Delineamos rutas para la síntesis dirigida al sitio de 10 chalconas diferentes con su correspondiente análisis de RMN.

La cepa S. mutans UA159 se cultivó en medio BHI y los inóculos de reserva bacteriana se establecieron en la escala de McFarland 0.1-0.08. Los experimentos se llevaron a cabo en una microplaca de 96 pocillos y posteriormente se analizaron utilizando mediciones automáticas de absorbancia del lector de placas y un ensayo de viabilidad de resazurina. Inicialmente, se evaluaron 10 chalconas tratando $S$. mutans con concentraciones de dilución en serie (1 mM-0.0078 mM). En un segundo experimento, se analizó la chalcona 12 y se usó un ensayo de cinética de tiempo muerto para determinar el efecto de exposición al tiempo del fármaco. El control positivo para los experimentos fue clorhexidina al $0,12 \%$. Obtuvimos biofilms maduros de 3 días usando glucosa como adyuvante para observar posibles efectos inhibidores de biofilm. Se utilizó el análisis estadístico no paramétrico considerando el patrón gaussiano de distribución de datos y $p<0.05$. Se observó para el primer experimento que chalcona 12 tenía una actividad inhibitoria mínima a concentraciones de $250 \mathrm{nM}$. En el segundo experimento, se observó una actividad bacteriostática para este nuevo compuesto asociado con un perfil de crecimiento más lento en comparación con el grupo de vehículos durante las primeras tres horas. El último experimento no demostró diferencias en la formación de biofilm entre chalcona 12 y los grupos no tratados, explicándose como un protocolo de exposición insuficiente al fármaco. Nuestros hallazgos demostraron que chalcona 12 tiene efectos bacteriostáticos prometedores que funcionan en concentraciones nanomolares contra S. mutans. 


\section{Trabajos presentados en modalidad de panel}




\section{Beneficios del licopeno en tratamiento de liquen plano erosivo oral con displasia leve a moderada}

\author{
Claudia López de Ayala', Ariely Marihual', Marcela Correa' \\ ${ }^{1}$ Facultad de Odontología, Universidad Mayor. Temuco, Chile.
}

Introducción: El liquen plano erosivo oral es una enfermedad inflamatoria crónica de base inmunológica. La importancia de conocerlo se fundamenta en que se describe como un trastorno potencialmente maligno, especialmente cuando se añaden factores irritativos como el tabaco. ${ }^{1,2}$

Reporte del Caso: Paciente mujer de 41 años, ASA I, acude a centro diagnóstico de la Universidad Mayor sede Temuco derivada para evaluación de lesiones reticulares y erosivas en cara interna de ambas mejillas, indoloras, de un año de evolución. Relata consumir 10 cigarrillos diarios hace 25 años.

Se realiza biopsia incisional de ambas lesiones. Se encuentran hallazgos de liquen plano con displasia leve a moderada. Como tratamiento inicial se indica Clobetasol, vitamina $A$ y vitamina $E$ en plastibase de uso tópico y se solicita la eliminación del hábito tabáquico. Al control en 6 meses se aplica azul de toluidina y se biopsia la zona más teñida obteniendo el mismo diagnóstico histopatológico anterior. Se indica iniciar tratamiento con licopeno 10 mg en comprimidos por 3 meses, donde se obtiene una evidente mejoría clínica de las lesiones.

Discusión: El licopeno tiene efectos contra cáncer de mama, cérvix, pulmón, cavidad oral y próstata. Así, Konijeti et al. realizaron un estudio en ratones donde compararon el efecto de licopeno en comprimidos y una dieta control. Encontraron que los ratones alimentados con licopeno presentaron menor incidencia de cáncer y menor daño oxidativo al ADN que las del grupo control. Contribuyendo a la reparación del ADN e inhibiendo cambios displásicos, estudios describen su capacidad para revertir lesiones precancerosas y actuar sobre el desarrollo del cáncer oral. ${ }^{4,5}$

Conclusión: En este caso se obtuvo una considerable remisión de las lesiones tratadas con licopeno, lo cual determina la importancia de indicar a los pacientes de que fuentes adquirir antioxidantes y utilizar sus beneficios preventivos e interceptivos ante lesiones orales premalignas.

\section{Linfoma no Hodgkin primario de paladar duro en paciente embarazada: reporte de caso}

Natalia Zúñiga' ${ }^{1}$ Valentina Aguilera ${ }^{1}$, Alejandra Castro ${ }^{1}$

'Escuela de Odontología, Facultad de Medicina, Pontificia Universidad Católica de Chile. Santiago, Chile.

Introducción: Los linfomas son un grupo heterogéneo de neoplasias malignas, producidas principalmente en ganglios linfáticos. Sin embargo, pueden tener origen extraganglionar siendo mucho más frecuente en linfomas no Hodgkin (LNH) que en los de Hodgkin. De todos los linfomas extraganglionares, solo $2 \%$ afecta la cavidad oral de forma primaria. El propósito de este reporte es presentar un caso de LNH primario localizado en paladar duro en una mujer embarazada y describir la presentación clínica e histopatología observada.

Reporte de caso: Se presenta un caso clínico de una paciente de sexo femenino, peruana, de 40 años, sin antecedentes mórbidos relevantes, que cursa un embarazo de 20 semanas de gestación. Consulta por aumento de volumen palatino de un mes y medio de evolución, indoloro, sin fiebre, baja de peso o adenopatías. Al examen intraoral destaca desdentada parcial bimaxilar, enfermedad periodontal, policaries; y extensa tumoración de consistencia firme, superficie lobulada, límites definidos, cubierta por mucosa de aspecto normal, extendiéndose en el hemipaladar izquierdo, sin causar movilidad dentaria. Se realiza biopsia incisional de la lesión. El estudio histopatológico mostró epitelio plano pluriestratificado normal. Desde corion en adelante, abundante infiltrado linfocitario, casi 100\% CD20+, que infiltra tejido glandular, persistiendo algunos islotes epiteliales, con mitosis atípicas, células de núcleos pequeños, hipercromáticos, mezclados con algunas células grandes de citoplasma escaso y nucléolo prominente. Las células fueron casi 100\% BCL2+ y 20-30\% Ki67+. Los hallazgos fueron compatibles con linfoma difuso de células $B$ grandes (DLBCL).

Conclusión: El compromiso extraganglionar del DLBCL es frecuente y virtualmente puede afectar cualquier tejido, siendo lo más habitual el tracto gastrointestinal. Opuestamente, la afección palatina como sitio primario es un hallazgo muy infrecuente que se observa en escasos reportes de la literatura. 


\section{Manejo oral previo a tratamiento con inhibidor de RANK-L en paciente con compromiso sistémico múltiple}

\author{
María Francisca Cortés ${ }^{1}$, Nicolás Santander ${ }^{1}$, Yelitza Niño ${ }^{1}$
}

'Escuela de Odontología, Facultad de Medicina, Pontificia Universidad Católica de Chile. Santiago, Chile.

Introducción: El tratamiento con inhibidores de RANK-L, como la administración endovenosa de Denosumab, ha sido considerado efectivo en patologías óseas como la osteoporosis. A pesar de su adecuado perfil de seguridad, este medicamento sigue inspirando preocupación por sus efectos a largo plazo incluso posteriores al cese de la administración. Dentro de ellos se incluyen eventos dermatológicos severos, infecciones graves y osteonecrosis de los maxilares por medicamentos, siendo este último el más grave y de mayor relevancia para los odontólogos.

Reporte de caso: Acude a evaluación en la Clínica Odontológica Docente UC paciente de sexo femenino, 53 años, por dolor en zona anteroinferior izquierda. En su anamnesis remota relata múltiples alergias (Penicilinas, Quinolonas, Dipirona, Fluoxetina, Fentanyl, Torecan y Goma Sántica), enfermedades sistémicas (Esclerodermia, Síndrome de Sjögren, Hipotiroidismo, Reflujo Gastroesofágico, Osteoporosis y Fenómeno de Raynaud) y consumo de medicamentos (Eutirox, Esomeprazol, VitD, Calcio y colirio oftálmico). Además, cuenta con indicación médica de terapia endovenosa con Denosumab por osteoporosis, pero que aún no inicia por motivos económicos. Al examen físico extraoral presenta fascie hipotiroidea, pérdida de la cola de la ceja, piel reseca y acartonada, cantidad reducida de pelos, telangectasias y pseudoprognatismo por desdentamiento parcial superior. Intraoralmente se aprecian múltiples pérdidas dentarias, restos radiculares, pérdida de estabilidad y soporte oclusal posterior. Radiográficamente presenta una reabsorción ósea marginal horizontal avanzada y múltiples lesiones osteolíticas radiculares. Se realiza interconsulta con médicos tratantes por consideraciones para la atención, administración de profilaxis antibiótica (Claritromicina en suspensión oral), exodoncia de los 18 dientes remanentes por riesgo de generar osteonecrosis por medicamentos y confección de prótesis de removible superior e inferior previo al inicio de terapia con Denosumab.

Discusión y conclusión: Los odontólogos deben conocer el riesgo de desarrollar osteonecrosis por medicamentos en relación con la administración de Denosumab para prevenir y controlar los factores de riesgo orales antes del inicio de la terapia. También se recomienda evitar procedimientos dentales invasivos durante su administración. Además, el trabajo interdisciplinario y la comunicación efectiva con el paciente son herramientas primordiales para un desenlace favorable. Finalmente, el trabajo coordinado entre todas las partes permitirá reducir los efectos adversos de su consumo.

\section{Provisionalización inmediata adhesiva en sector anterior: Diente natural cómo póntico. Reporte de caso clínico}

Elías Coroseo ${ }^{1}$, Antonio Alvear ${ }^{1}$, Claudio Jorquera $^{1}$

'Escuela de Odontología, Facultad de Medicina, Pontificia Universidad Católica de Chile. Santiago, Chile.

Introducción: La pérdida de dientes en el sector anterior implica una importante preocupación por parte del paciente sobre la recuperación de la estética. Es fundamental el manejo de provisorios que la recuperen durante la reparación tisular y planificación de la rehabilitación definitiva. El desarrollo de tecnologías adhesivas y resinas compuestas más resistentes permite otorgar una solución inmediata, conservadora, económica y estética.

Reporte de caso: Paciente de 47 años, ASA II (HTA). Consulta por estética en diente anterosuperior. Manifiesta preocupación económica respecto a las opciones de tratamiento. Presenta dentición completa. Diente 1.2 con recesión gingival (tipo 2 de Cairo) y movilidad grado 3. Diagnóstico de lesión endoperiodontal verdadera con pronóstico periodontal hopeless. En una única sesión, se realiza extracción y decoronación de diente 1.2, limpieza y remoción de caries. Se reconstruye con resina compuesta, aumentando ancho mesio-distal y otorgando terminación de póntico ovalado. Se feruliza por palatino a dientes 1.1 y 1.3 combinando alambre de ortodoncia trenzado en cervical y resina compuesta en tercio incisal.

Discusión/conclusión: Adherir materiales restauradores en la cara palatina incisal al punto de contacto con el antagonista puede producir interferencias en apertura. En este caso, el overjet y overbite aumentados permitió aumentar el grosor de la banda de resina compuesta usada para estabilizar el póntico, mejorando su resistencia a la flexión.

El uso de la corona del diente extraído cómo póntico permite una fácil manipulación clínica y lograr un resultado estético. Se entregó una solución inmediata y mínimamente invasiva en dientes vecinos. Paciente se manifestó altamente satisfecha.

\section{Regeneración endodóntica en diente con antecedente de Dens evaginatus. Reporte de Caso}

Luisa Barrón', Alejandra Salinas ${ }^{1}$

'Escuela de Odontología, Facultad de Medicina, Pontificia Universidad Católica de Chile. Santiago, Chile. 
Introducción: La pérdida de vitalidad en dientes permanentes inmaduros ocurre generalmente como consecuencia de trauma y caries, menos frecuente asociada a malformaciones como el Dens Evaginatus, que se manifiesta como una cúspide extra o protrusión de tejido que queda generalmente en trauma oclusal dejando susceptible al tejido pulpar a quedar expuesto y desvitalizarse. Cuando esto ocurre, debe considerarse tratar con Inducción al cierre apical o mediante Procedimiento endodóntico regenerativo (REP). La ventaja de este último es que, además de resolver la infección endodóntica, ocupa los principios de la ingeniería de tejidos promoviendo el desarrollo radicular. El área de la Endodoncia regenerativa requiere documentar estos casos en divulgaciones científicas y ojalá colaborar con la base de datos de Asociación Americana de Endodoncia (AAE) para establecer las mejores prácticas en el tratamiento e investigación.

Reporte de caso: Presentamos el caso de un paciente de sexo masculino de 10 años derivado a Clínica San Carlos para una segunda opinión después haber acudido de urgencia a otro servicio. Se Diagnóstica diente 4.5 terapia previamente iniciada con antecedente de Dens evaginatus. Esto se constata con la radiografía que remite su odontóloga tratante y el antecedente de la familia quienes identifican que la malformación es igual a la del diente homólogo aún vital. Se le solicitó CBCT que muestra escaso desarrollo radicular y se planifica REP siguiendo las recomendaciones de la AAE que compatibilizan la desinfección del canal radicular, mínimo daño a las células madres de la papila apical y prevención de complicaciones estéticas asociadas a biomateriales. Se documenta el seguimiento del caso a 5 meses logrando ausencia de signos y síntomas, formación de tejido duro al interior del canal e indicio de cierre apical. En este plazo esta técnica ha dado resultados promisorios, pero es necesario el seguimiento a largo plazo para detectar posibles complicaciones.

\section{Manejo clínico endodóntico de secuela de trauma en diente permanente inmaduro. Reporte de caso}

\author{
María José Aguila' ${ }^{1}$ Catalina Toledo', Josefina Retamal' \\ 'Escuela de Odontología, Facultad de Medicina, Pontificia Universidad \\ Católica de Chile. Santiago, Chile.
}

Introducción: El trauma dentoalveolar (TDA) corresponde a un conjunto de lesiones que comprometen al diente y sus estructuras de soporte, constituyendo un gran problema de salud pública por su alta incidencia, manejo clínico y complicaciones. La necrosis pulpar es una secuela frecuente y cuando sucede en un diente permanente inmaduro con ápice abierto, es necesario realizar una técnica de apexificación. El agregado de trióxido de mineral (MTA) es ampliamente utilizado como tope apical por sus excelentes características. Diversos estudios han demostrado que otorga un menor tiempo de reparación ósea en comparación con otros materiales.

Reporte de caso: Paciente 23 años, ASA I. Al acudir a control dental, se observa cambio de coloración coronario y fístula en relación a diente 2.1, con antecedente de TDA infantil tratado en Haití. Se realizan test de sensibilidad, radiografía periapical, y fistulografía. Diagnóstico: Absceso apical crónico. Al análisis radiográfico se observa canal amplio con cierre apical incompleto y área radiolúcida. Se realiza necropulpectomía y tope apical de MTA utilizando microscopía. Para obturar se fabrica cono maestro individualizado, uniendo dos conos de gutapercha \#80. Radiografía de control de obturación. Control clínico a la semana. Control clínico y radiográfico a los 6 meses, observando reparación de la lesión apical.

Discusión: Los criterios de éxito del tratamiento endodóntico incluyen la resolución de los cambios inflamatorios periradiculares, ausencia de sintomatología y cambios radiográficos de reparación ósea. Los signos clínicos y radiográficos observados en el paciente a los 6 meses evidencian un resultado exitoso del tratamiento. El uso de MTA incluye recubrimiento directo, reparación de perforaciones, apexificación y formación de barrera en dientes con ápices abiertos. Esta última propiedad, permitió observar la formación de trabeculado óseo a los 6 meses de control y su eficacia en el tratamiento de estos dientes, similar a los casos reportados en la literatura.

\section{Manejo de paciente con dentición severamente desgastada con protocolo de "Three Step" modificado: reporte de un caso}

Josefina Cid' ', Javiera Rojas', Bastián Guzmán', Beatriz Mellado

'Escuela de Odontología, Facultad de Medicina, Pontificia Universidad Católica de Chile. Santiago, Chile.

Introducción: La erosión dental, se define como la pérdida irreversible de estructura dentaria debido a una disolución química provocada por ácidos, sin presentar un origen bacteriano. Su etiología es multifactorial y su prevalencia oscila entre un $1,8 \%$ a un $34,1 \%$ de la población. Por medio de la observación clínica de desgastes de dientes anterosuperiores, se estableció la "clasificación de erosión clínica anterior" (ACE), la cual tiene 6 categorías en orden ascendente de pérdida de estructuras dentarias. Se ha sugerido la técnica de "Three Step", para el tratamiento rehabilitador de paciente con desgaste severo, ya que permite 
preservar mayor estructura dentaria, se evita realizar tratamientos endodónticos electivos y el resultado estético es superior y predecible para el paciente. Objetivos: Mostrar la rehabilitación con protocolo de "Three Step" modificado en su fase 1, en paciente con clasificación ACE V.

Reporte de Caso: Acude a internado de Rehabilitación Oral paciente de 49 años, ASA I, consultando por opciones de rehabilitación en el sector anterior luego de múltiples fracasos. Paciente relata dieta altamente acidogénica y sensación de apriete dentario. Clínicamente se observa ausencia de enfermedad periodontal. Piezas anterosuperiores severamente desgastadas, clasificación ACEV y exposición de dentina oclusal. Se opta por rehabilitación mediante técnica de "Three Step", modificando la fase 1, instalando plano oclusal de cobertura total superior para la toma de consciencia sobre el mal hábito de apriete dentario. Se realiza montaje de articulador, aumento de dimensión vertical (DVO) arbitraria en articulador y traspaso a plano oclusal para manejo de DVO intraoral. Realización de encerado dentario en modelos y prueba en boca. Se continúa devolviendo el soporte posterior, utilizando llaves de silicona y resinas termo plastificadas, para luego devolver la guía anterior con la rehabilitación definitiva del sector anterosuperior mediante prótesis fijas unitarias y carillas

\section{Prótesis Fija Unitaria en Dientes con Microrrizosis: Reporte de Caso}

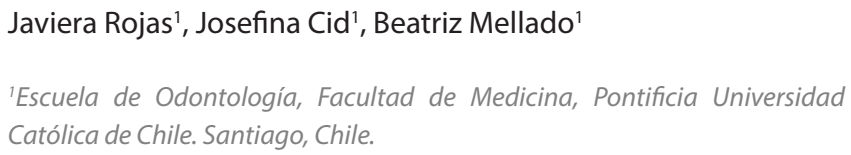

Introducción: En la actualidad restaurar el sector anterior supone un problema añadido a nuestra práctica diaria, ya que cada día aumentan aún más las exigencias estéticas. En muchas ocasiones, además, se añade la necesidad de ferulizar dichas restauraciones, ya sea por anomalías dentarias, problemas ortodóncicos o periodontales.

Una de las alteraciones a las que el dentista se puede ver expuesto es la microrrizosis: anomalía donde la altura de la raíz de un diente es menor que la altura de su corona; es una condición de baja prevalencia que puede ser causada por trauma, radiación o alteración en el desarrollo.

El objetivo del presente caso es describir el tratamiento rehabilitador del sector anterosuperior en un paciente con alteraciones estéticas y un diente con microrrizosis.

Reporte de Caso: Consulta al internado de Rehabilitación Oral paciente de 49 años, ASA I, por inconformidad estética. Al examen intraoral se observan piezas anterosuperiores con múltiples restauraciones de resina compuesta. Al examen radiográfico se visualiza reabsorción ósea marginal horizontal marcada y microrrizosis de diente 2.1. Se propuso como plan de tratamiento realizar terapia periodontal y la restauración estética del diente 1.1, 2.1 y 2.2 con prótesis fija plural a férula.

Discusión: Desde tiempos remotos, la relación mínima que debe existir entre la corona clínica y la raíz soportada por tejido óseo es idealmente 2:3, pero se exige como mínimo 1:1. No obstante, al verse enfrentado ante una relación corono-radicular desfavorable ésta no impide que podamos realizar un tratamiento altamente estético; por ende, es ahí donde juega un papel fundamental una de las funciones de las prótesis fijas plurales: ferulizar. No obstante, es requisito el control de la enfermedad periodontal para poder lograr resultados óptimos.

Conclusión. Una correcta planificación de tratamiento debe ser individualizada y ejecutada con el fin de atender las necesidades de cada paciente, realizando una adecuada secuencia de tratamiento.

\section{Insuficiencia suprarrenal en polineuropatía desmielinizante inflamatoria crónica. Reporte de caso}

Dusan Marinkovic ${ }^{1}$, Carla Madrid', Francisco Pinedo'

'Escuela de Odontología, Facultad de Medicina, Pontificia Universidad Católica de Chile. Santiago, Chile.

Introducción: La Polineuropatía desmielinizante inflamatoria crónica (CIDP) corresponde a un trastorno neurológico que afecta al sistema nervioso periférico, caracterizado por una debilidad muscular y sensorial progresiva en las extremidades, de manera continua por un tiempo mayor a dos meses. Tiene carácter autoinmune. Su prevalencia se estima en 0.2-0.5 casos por cada 100.000 habitantes. Puede manifestarse a cualquier edad y sin predilección por sexo, pero tiene mayor predominio en la quinta y sexta década. Su etiología aún es incierta, en la mayoría de los casos reportados se dice que es idiopática. El diagnóstico suele ser clínico junto a un examen electrofisiológico. El tratamiento se basa en corticoterapia, inmunoterapia, plasmaféresis e inmunosupresores.

Reporte de Caso: Al curso de cirugía bucal acude un paciente de 60 años, sexo masculino, con interconsulta de rehabilitador para exodoncia de dientes remanentes en mal estado. Relata ser portador de CIDP diagnosticada el 2006, que debutó súbitamente con síntomas motores y sensoriales por un brote infeccioso de etiología desconocida, además de dolor neuropático asociado. A 
la fecha se encontraba en terapia farmacológica con prednisona, tramadol, paracetamol y omeprazol. Al examen físico se observa una marcha hemiparética, fascies cushingoide, acropaquia, onicomicosis, hipertensión arterial (HTA) y hemianopsia homónima homolateral izquierda. Dada los antecedentes y características clínicas, se sospecha de una insuficiencia suprarrenal asociada a corticoterapia crónica, se realiza interconsulta a médico tratante por HTA e insuficiencia suprarrenal. Paciente fue controlado por médico, se ajusto dosis de corticoides y comenzó tratamiento antihipertensivo. Para el manejo quirúrgico se prescribió profilaxis antibiótica.

Conclusión: La integración de los diferentes signos y sintomas se presenta como el principal desafío en la atención dental, como también derivar de manera oportuna frente a la sospecha de patologías concomitantes. Frente a un paciente que presenta corticoterapia crónica en presencia de signos característicos, es importante considerar la posibilidad de insuficiencia suprarrenal.

\section{Reparación de una Periodontitis Apical Asintomática, reporte de un caso clínico}

Fabián Iturbe ${ }^{1}$, Carla Richaud ${ }^{1}$, Ivana Vásquez¹ , Mónica Arce

'Escuela de Odontología, Facultad de Medicina, Pontificia Universidad Católica de Chile. Santiago, Chile.

Introducción: La Periodontitis Apical Asintomática se define como la inflamación y destrucción de los tejidos perirradiculares como consecuencia de una etiología bacteriana que comienzan en el órgano pulpary se propaga a los canales radiculares del diente. El objetivo del tratamiento endodóntico es reducir al mínimo la carga bacteriana presente mediante una terapia químico-mecánica. La activación con ultrasonido mejora tanto los aspectos mecánicos como químicos del procedimiento de irrigación, el cual tiene por objetivo remover microorganismos, barro dentinario y detritus que no pudo ser quitado por la instrumentación ni la irrigación pasiva. Por lo tanto, se ha visto que la activación del irrigante con ultrasonido puede mejorar la efectividad de la terapia endodóntica. La finalidad del tratamiento de una patología periapical es lograr que estos tejidos sanen y se logre una reparación a este nivel. Por esto se debe realizar un seguimiento clínico radiográfico regular, debido a que el tratamiento de endodoncia no finaliza con la OBSCR. A continuación, se presenta el caso de una paciente género femenino, 57 años, ASA 1 que se presentó con diente 1.2 con el diagnóstico de Periodontitis Apical Asintomática con Terapia Previamente Iniciada, hace 2 años expuesta al medio bucal. Se emplearon limas manuales $\mathrm{K}$, irrigación con NaOCl al 5.25\%. Medicación intracanal con $\mathrm{Ca}(\mathrm{OH}) 2$ en formato Ultracal durante 7 días. Previo al protocolo de Irrigación Final se activó el Hipoclorito de Sodio con Ultrasonido. Conos gutapercha al $2 \%$ y cemento TopSeal con técnica compactación lateral en frío.

Objetivo: Evaluar proceso de reparación apical posterior al tratamiento endodóntico

Metodología: Evaluación radiográfica comparativa de control de obturación, al 1er, 2do y 3er año luego de la alta clínica del tratamiento endodóntico.

Resultados: Mediante los controles radiográficos es posible evidenciar una disminución del diámetro y la radiolucidez de la lesión, observando trabeculado óseo y reparación de la zona.

\section{Síndrome $\mathrm{C}$ de Opitz con un Alcance Odontológico: Revisión Bibliográfica y Reporte de Caso}

María Eugenia Borja' , Cristina Bofarull', Carla Mendieta'

'Escuela de Odontología, Facultad de Medicina, Pontificia Universidad Católica de Chile. Santiago, Chile.

Antecedentes. El Síndrome Trigonocefalia $C$ de Opitz es un trastorno genético poco común y heterogéneo con una tasa de mortalidad cercana al 50\% dentro del primer año de vida. Su principal característica es la sinostosis de la sutura metópica, asociada a anomalías craneofaciales, facies inusuales, rebordes alveolares anchos y frenillos orales múltiples; anomalías sistémicas como defectos en las extremidades y articulaciones, anomalías viscerales, del sistema nervioso central, cardiacas y renales, piel extremadamente elástica, discapacidad psicomotora e hipotonía. Su etiología genética se ha asociado a mutaciones en el gen CD96 con herencia autosómica recesiva, pero también se sugiere en algunos casos herencia autosómica dominante. En el contexto de atención odontológica de pacientes con necesidades especiales, se presenta el caso de una paciente portadora de Síndrome C, sexo femenino de 47 años.

Objetivo. Esta revisión bibliográfica y reporte de caso tiene por objetivo revisar la evidencia respecto al Síndrome Trigonocefalia C 
de Opitz, específicamente sobre sus manifestaciones craneofaciales e implicancias odontológicas, aplicando estos parámetros al caso clínico de la paciente.

Método. Se realizó una búsqueda amplia en la base de datos PubMed y Google Scholar con los términos "Opitz Trigonocephaly Syndrome”, "Opitz C Syndrome” ${ }^{\text {" }}$ Opitz C Trigonocephaly Syndrome". Los criterios de inclusión fueron: reportes de casos en inglés con título y resumen relacionados con el tema de la revisión. No se acotó la búsqueda por año de estudio. Se obtuvo 13 artículos en la búsqueda de PubMed de los cuales se seleccionó 6 . Dentro de las referencias de estas publicaciones, se seleccionó 5 trabajos más. En Google Scholar se encontró un total de 696 estudios, de los cuales se seleccionó 4, obteniendo finalmente 15 artículos para esta revisión.

\section{Carcinoma espinocelular en la cavidad oral, la importancia de un diagnóstico temprano}

\author{
Camila Boin" Matilde del Olmo ${ }^{1}$, Felipe Lillo² \\ ${ }^{1}$ Facultad de Odontología, Universidad de Chile. Santiago, Chile. ${ }^{2}$ Servicio de \\ Patología Oral, Hospital San Juan de Dios, Santiago, Chile.
}

Introducción. El carcinoma oral de células escamosas (COCE) es la neoplasia maligna más frecuente de la cavidad oral, siendo una amenaza para la salud pública mundial. De etiología multifactorial, asociada a factores de riesgo como tabaco, alcohol y edad mayor a 45 años. Su apariencia clínica varía desde lesiones leucoplásicas y eritroplásicas en estadios tempranos hasta masas exofíticas o úlceras que no cicatrizan en etapas más avanzadas. El diagnóstico temprano es difícil, la mayoría de las lesiones tienden a ser asintomáticas, siendo el tratamiento más complicado y de peor pronóstico. El objetivo de este trabajo es mostrar la importancia de un diagnóstico precoz de CEC en cavidad oral.

Reporte de Caso. Paciente, 56 años, sexo masculino, diabetes mellitus, acude a Patología Oral del Hospital San Juan de Dios derivado desde atención primaria por úlcera oral de 1 año de evolución. Al examen clínico se observa úlcera en zona retromolar derecha, de $2 \times 1.5 \mathrm{~cm}$, bordes indurados con eritroplasia. Se palpa linfadenopatía cervical derecha, inmóvil, dura y adherida a planos. Se solicita realizar biopsia urgentemente, diagnosticando un CEC. Al scanner se observa invasión del tejido óseo.

Discusión. EI CEC en la cavidad oral tiene características que difieren de otras neoplasias malignas. Esta zona presenta interacciones entre tejido óseo, músculos, dientes y piel que no son comparables con ninguna otra región anatómica, generando secuelas psicosociales que afectan negativamente la calidad de vida del paciente. Además, el COCE se caracteriza por una alta tasa de metástasis cervical e invasión local, la que afecta directamente al pronóstico de los pacientes.

Conclusión. Es fundamental diagnosticar y realizar las derivaciones correspondientes para que el paciente reciba un tratamiento adecuado en el menor tiempo posible. Esto podría mejorar el pronóstico y además evitaría que la neoplasia invada más tejidos logrando una mejor calidad de vida para el paciente.

\section{El desafío diagnóstico que implican las patologías vesiculoampollares autoinmunes en la cavidad oral}

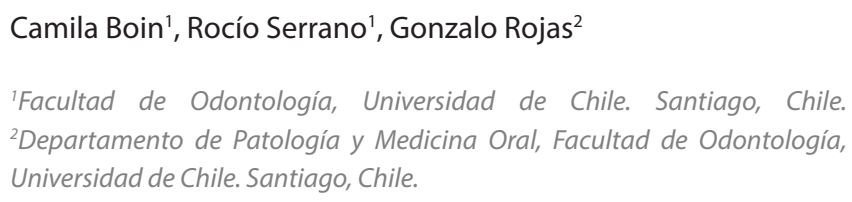

Introducción: El Penfigoide de las Membranas Mucosas (PMM) y el Pénfigo Vulgar (PV) son enfermedades ampollares mucocutáneas autoinmunes. La mucosa más afectada es la oral, pero puede comprometer la ocular, esófago y laringe. Ambas comparten características clínicas constituyendo un desafío diagnóstico, indispensable para su tratamiento. El objetivo de este trabajo es presentar el manejo clínico de un caso con lesiones que comprometen severamente la mucosa oral, cuyo diagnóstico diferencial clínico es complejo.

Reporte de caso: Mujer, 48 años, asiste a la clínica de Medicina Oral del Hospital Clínico Universidad de Chile derivada desde Inmunología por presentar lesiones orales importantes. En los últimos tres meses, el cuadro ha sido severo con dolor intenso, disfagia y significativa pérdida de peso. Al examen clínico presentó ulceras extensas en labio inferior, cara interna de mejillas, piso de boca y paladar blando, con irritación de la conjuntiva ocular. Signo de Nikolsky negativo.

Se indicaron corticoides (Prednisona $40 \mathrm{mg} /$ día), antisépticos, antimicóticos (Miconazol 2,2\%) y colutorios, para disminuir la sintomatología. Se dio una interconsulta a Oftalmología.

A los 15 días, presentó aumento del número de lesiones y signo de Nikolsky positivo. Se cambió esquema terapéutico, aumentando la dosis de corticoides (60 mg/día) e indicando un fármaco inmunosupresor tópico (Tacrolimus 0,1\%). A la semana el cuadro disminuyó significativamente. 
Discusión: El diagnóstico diferencial entre PMM y PV es complejo. La biopsia es fundamental para el diagnóstico definitivo, pero realizarla en un estado agudo complejiza el procedimiento quirúrgico y la sintomatología del paciente. El retraso de la instauración del tratamiento adecuado puede empeorar el cuadro comprometiendo otros tejidos. En caso de compromiso ocular, la interconsulta a oftalmología es mandatorio.

Conclusión: El tratamiento es similar para ambas patologías cuando las lesiones son orales, usando corticoides sistémicos y locales con control de infección. El caso presentado prioriza disminuir el cuadro para planificar biopsia y confirmar diagnóstico.

\section{Quistes y tumores odontogénicos más prevalentes en relación a terceros molares impactados}

Catalina Borroni', Consuelo Zamorano', Solange Barraza', Verónica Palacios ${ }^{1}$

'Escuela de Odontología, Facultad de Medicina, Pontificia Universidad Católica de Chile. Santiago, Chile.

Antecedentes: La prevalencia global de quistes y tumores odontogénicos asociados a terceros molares impactados es de un 5.3 $\%$. Los quistes y tumores más prevalentes son el quiste dentígero, queratoquiste odontogénico y ameloblastoma. Las lesiones anteriormente mencionadas son afecciones no inflamatorias que están fuertemente relacionadas en su mayoría con terceros molares impactados.

Pregunta: ¿Cuál es la frecuencia de quistes del desarrollo y tumores odontogénicos relacionados a terceros molares impactados?

Objetivo general: Caracterizar los quistes y tumores odontogénicos más prevalentes en relación a terceros molares impactados.

Objetivos específicos: 1) Indagar sobre quistes y tumores odontogénicos más prevalentes en terceros molares impactados; 2) Describir las características clínicas, radiológicas e histológicas de los quistes y tumores odontogénicos más prevalentes en relación a terceros molares impactados; 3) Analizar la prevalencia de quistes y tumores odontogénicos en relación a terceros molares dado la importancia como motivo de consulta de la Patología vinculada a estos molares.

Métodos: Para esta revisión narrativa, se analizaron diversos estudios obtenidos desde los buscadores PubMed, Dental \& Oral Sciences Source y Medline, realizando la búsqueda con los MeSH "cyst", "tumor", "third molar", "neoplasms", "odontogenic tumor", "odontogenic cyst". "dentigerous cyst", "odontogenic keratocyst", "ameloblastoma". Los criterios de inclusión utilizados fueron todas aquellas revisiones sistemáticas disponibles en línea y de forma gratuita y que tengan relación con quistes y tumores de terceros molares. Los criterios de exclusión fueron estudios de una longevidad mayor a 5 años y otro idioma que no sea inglés o español.

\section{Atenciones odontológicas domiciliarias: implicancias para las enfermedades cognitivas del adulto mayor con dependencia severa}

Natalia Álvarez', Karin Arriaza', Gabriela Contreras' ${ }^{1}$ Tiare León'1, Ignacia Ríos', Karla Ríos', Macarena Toro', Duniel Ortuño

'Escuela de Odontología, Facultad de Medicina, Pontificia Universidad Católica de Chile. Santiago, Chile

Resumen: La población de adultos mayores ha aumentado en Chile y el mundo, y junto con ello la prevalencia de enfermedades cognitivas. Estudios observacionales han sugerido que las atenciones odontológicas podrían disminuir el daño cognitivo en adultos mayores en situaciones de dependencia severa. Se realizó una revisión no sistemática con el objetivo de evaluar el efecto de las atenciones dentales durante visitas domiciliarias, en el pronóstico de enfermedades cognitivas en pacientes adultos mayores en situación de dependencia severa. La búsqueda fue realizada por tres revisores en las bases de datos Pubmed y Lilacs. Después de aplicar los criterios de selección de estudios se analizaron tres artículos primarios.

Se encontró asociación entre la pérdida de piezas dentarias y el deterioro de la función cognitiva en adultos mayores, mientras que la cobertura odontológica se asoció con un progreso a nivel cognitivo del adulto mayor en situación de dependencia severa. La certeza de la evidencia según los criterios GRADE fue baja, con una recomendación a favor del tratamiento domiciliario de estos pacientes.

Se concluyó que la atención domiciliaria mejora la salud oral en los pacientes adultos mayores con dependencia severa y por consiguiente mejora el pronóstico de las enfermedades cognitivas que se ven influidas por la calidad masticatoria. Se sugiere aumentar la cobertura nacional de la atención odontológica domiciliaria, establecida durante el año 2019 como componente del Programa Odontológico Integral en Chile y monitorear el impacto de las intervenciones en la vida de los pacientes en esta situación. 


\section{Revisión sistemática sobre acceso, información y adaptación del sistema de salud para migrantes en Chile}

\author{
Kathya Guerrero ${ }^{1}$, Lissette Betancourt ${ }^{1}$, María Bravo', Carla \\ Latorre' ${ }^{1}$ Claudia Véliz' \\ 'Escuela de Odontología, Facultad de Medicina, Pontificia Universidad \\ Católica de Chile. Santiago, Chile
}

En Chile, la migración ha aumentado aceleradamente en los últimos años. La vulnerabilidad social como es la situación irregular de documentación, pobreza, malas condiciones de vivienda, desempleo o empleo informal, procesos de discriminación y abuso, predispone al deterioro de su salud. Por lo tanto, adaptar el sistema de salud frente a estas nuevas condiciones es el desafío que enfrenta Chile.

El objetivo de esta revisión sistemática es contestar la pregunta ¿Cuáles son las políticas públicas exitosas para adaptar los sistemas de salud a los migrantes?

Para responderla se utilizó el buscador PubMed.

Como resultado se muestra que en Chile se han implementado programas enfocados en abordar el proceso migratorio, tanto por parte del personal de salud como por el sistema público en general, además del planteamiento de ocho grandes objetivos por parte del Ministerio de Salud enfocados en garantizar el acceso equitativo al sistema.

Los principales resultados muestran que las falencias están en acceso, información y adaptación cultural. La experiencia internacional incluye propuestas como desarrollo de planes para facilitar el acceso y mejorar la calidad de los sistemas de salud, entrega de información a prestadores e instruir a los profesionales.

Se concluye que el acceso a salud de migrantes debe enfocarse en implementar políticas sanitarias que impliquen integrar todo el proceso de ser migrante, adecuándose a la legislación y realidad de cada país. Se necesita seguimiento de las normativas para conocer la efectividad de ellas y aumentar la base de datos gubernamentales para impulsar políticas públicas adecuadas.

El proceso migratorio en Chile ha avanzado en la disminución de barreras y mejoramiento del acceso a salud, pero no lo ha hecho al ritmo adecuado, dejando evidentes falencias, por lo que es un desafío país continuar trabajando para brindar un buen servicio de salud a todos sus residentes.

\section{Consideraciones para el manejo de pacientes con Síndrome de Down durante la atención odontológica}

\author{
Sebastián Wall' María Francisca Cortés ${ }^{1}$, Carla Mendieta \\ 'Escuela de Odontología, Facultad de Medicina, Pontificia Universidad \\ Católica de Chile. Santiago, Chile
}

Introducción: El Síndrome de Down (SD) es una condición genética que posee manifestaciones tanto sistémicas como orales. En Chile, de 10.000 nacidos vivos 25 nacen con SD, situación mayor a la reportada en otros países de Latinoamérica. Si bien presentan una prevalencia de caries similar, mayor número de anomalías dentomaxilares y menor salud periodontal, evidencian una menor asistencia dental asociada a múltiples desafíos manifestados durante la atención odontológica.

Objetivo: Analizar la evidencia del último quinquenio sobre las consideraciones en el manejo que debe tener el odontólogo durante la atención dental de pacientes con SD.

Materiales y Métodos: Se efectuó una búsqueda amplia en las bases de datos PubMed y EBSCO con los términos clave "Down Syndrome", "Special needs", "Oral Health" y "Dental care access". Fueron incluidos los artículos publicados entre los años 2014-2019 en idioma inglés o español y excluidos los que no presentaban disponibilidad a texto completo. Se seleccionaron 18 artículos.

Resultados y discusión: Existe una falta de entrenamiento de los profesionales durante la atención de pacientes con SD en el uso de técnicas de adaptación, asociado a su condición y a los trastornos neuropsiquiátricos adicionales que presentan, como trastornos sensoriales y del espectro autista. Dado esto se sugiere considerar recomendaciones previas a la atención odontológica como el establecimiento de planes de acción claros, con una buena comunicación y la firma del consentimiento informado; y durante la atención, como el uso calmado de la voz, de la estabilización protectora y el fomento de las actividades preventivas.

Conclusión. Al brindar atención de salud oral a pacientes con SD es fundamental considerar para su manejo las comorbilidades asociadas que presentan, planificar la atención y perfeccionar el uso de técnicas de adaptación. Se requieren realizar estudios nacionales que proporcionen información de la situación actual de acceso y cobertura de la atención odontológica para pacientes con SD. 


\section{Medidas en Chile para superar barreras en atención de personas en situación de discapacidad}

\author{
Catalina Paz' ${ }^{1}$ Javiera Verdugo ${ }^{1}$, Cynthia Cantarutti ${ }^{1}$ \\ 'Escuela de Odontología, Facultad de Medicina, Pontificia Universidad \\ Católica de Chile. Santiago, Chile
}

Introducción. El 15\% de la población mundial padece algún tipo de discapacidad y actualmente la prevalencia ha ido en aumento debido al envejecimiento poblacional y a la presencia de enfermedades crónicas. Chile no es la excepción, donde el $20 \%$ de las personas son discapacitadas. Las personas en situación de discapacidad presentan mayor riesgo de presentar enfermedades bucodentales y además presentan dificultad en el acceso a la atención odontológica. Es por esto que es importante identificar los diferentes tipos de barreras y las medidas que se pueden tomar para enfrentarlas.

Pregunta. ¿Cuáles son las barreras de acceso a la atención de salud oral de las personas en situación de discapacidad y qué medidas se han implementado en Chile para solucionarlas?

Objetivo. Identificar las barreras de acceso a la atención de salud oral de las personas en situación de discapacidad y las medidas que se han implementado para solucionarlas en Chile.

Método. Se realizó una revisión narrativa, utilizando el buscador de datos PubMed, Tripdatabase y Google. Se incluyeron artículos disponibles a texto completo desde el 2014 en inglés, español y portugués.

\section{Aplicaciones de telemedicina para uso de pacientes con enfermedades crónicas. Revisión no sistemática}

Sofía Quezada', María Ignacia Reyes', Camila Rusque', María Paz Sapunar ${ }^{1}$, Mariana Valencia' ${ }^{1}$, Claudia Véliz

'Escuela de Odontología, Facultad de Medicina, Pontificia Universidad Católica de Chile. Santiago, Chile

Antecedentes: Las enfermedades crónicas afectan a miles de personas a lo largo del mundo, una alternativa que ha tenido éxito para ayudar a su prevención y control es la utilización de la Telemedicina, la que abarca cualquier actividad médica a distancia.

Objetivos: Analizar las distintas aplicaciones de la telemedicina utilizadas por pacientes con enfermedades crónicas. Métodos: Revisión narrativa. Se utilizaron bases de datos como PubMed, LILACS y sitios gubernamentales, utilizando los términos telemedicine, cronical disease, teleradiology, telerpathology, telemedicine/ classification/ methods, cardiology, DM, asthma, HIV.

Resultados: Se evaluaron 10 artículos que cumplieron con los criterios de inclusión. los artículos revisados analizan la aplicación de la telemedicina en enfermedades crónicas mediante: 1) programas online, 2) plataformas web, 3) cámaras, 4) teléfonos inteligentes, 5) videoconferencias, 6) monitoreos y 7) monitoreos complementados con programas online. Los ámbitos de acción de estas aplicaciones son principalmente la autogestión, la confirmación diagnóstica, la prevención de enfermedades en el paciente y el apoyo psicológico y social.

Conclusión: La telemedicina entrega información importante acerca de la autogestión y el autocuidado en pacientes con tratamientos de enfermedades crónicas, con el fin de mejorar la calidad de vida de aquellos sometidos a constantes monitoreos; permitiendo abordar los problemas con un enfoque centrado en el paciente, llegando más allá del bienestar físico, lo que hace que sea bien recibida por quienes la utilizaron. Al hacer uso de esta tecnología, se contribuye a una atención rápida en la salud hospitalaria, así como a una menor necesidad y frecuencia de visitas a los profesionales en los centros de atención de salud.

Palabras clave: Telemedicina, Enfermedades Crónicas, Aplicaciones Médicas Informáticas, Cardiología, Diabetes, Asma, VIH.

\section{Tratamiento conservador del Ameloblastoma, a propósito de un caso clínico}

Josefina Bendersky' ${ }^{1}$, Catalina Borroni' ${ }^{1}$ Luis Romo ${ }^{1}$

'Escuela de Odontología, Facultad de Medicina, Pontificia Universidad Católica de Chile. Santiago, Chile

Introducción: El Ameloblastoma es un tumor odontogénico benigno, desarrollado en la mandíbula (80\%) o en el maxilar. Existen tres tipos de acuerdo a sus características: uniquístico, multiquístico y periférico. El tratamiento del tumor es la cirugía, la cual puede ser radical; resección con márgenes de seguridad, o conservadora; enucleación, marsupialización o curetaje. Al tener alto porcentaje de recidiva, se recomienda el primer tipo de tratamiento mencionado, sin embargo, el análisis de distintas variables asociadas en el paciente en particular es mandatorio para la elección del tipo de tratamiento, ya que este implica grandes secuelas cosméticas y funcionales, requiriendo en algunos casos reconstrucción mediante injertos microvasculares.

Objetivo: Evaluar la evidencia para optar por un tratamiento conservador para el ameloblastoma en primera instancia, en base al análisis de las variables, en un caso clínico. 
Materiales y métodos: Se realiza una revisión narrativa a través de Pubmed, Cochrane Library, Google Scholary Epistemonikos, seleccionando 13 artículos de interés con criterios de inclusión y exclusión.

Reporte de caso: El paciente de sexo masculino, de iniciales JRS, 22 años, diagnosticado con Ameloblastoma uniquístico en el cuerpo mandibular derecho, tratado de manera conservadora, mediante enucleación y curetaje en mayo del 2017. Se ha realizado seguimiento hasta el día de hoy, sin signos de recidiva.

Discusión/Conclusión: Si bien la evidencia es controvertida, y a pesar de que el tratamiento conservador puede presentar mayores tasas de recurrencia, éste evita la disminución de la calidad de vida y las secuelas en el paciente, producto de la gran cirugía a la que pudiese ser sometido. Es relevante y una condición sin ecuanon el seguimiento del paciente para poder evaluar la posible recurrencia, que, si se presentara, se encontraría contenida en la mandíbula, probablemente en un foco discreto, más fácil de abordar quirúrgicamente y con menores secuelas para el paciente.

\section{Riesgo ocupacional por exposición a óxido nitroso en odontología}

\author{
Magdalena Raveau', María Eugenia Guevara' \\ 'Escuela de Odontología, Facultad de Medicina, Pontificia Universidad \\ Católica de Chile. Santiago, Chile
}

Introducción: La Academia Americana de Odontología Pediátrica (AAPD) reconoce la inhalación de óxido nitroso como un método seguro y una técnica efectiva para reducir la ansiedad, producir analgesia, y mejorar la comunicación efectiva con el paciente. Sin embargo, reconoce que la exposición al óxido nitroso ambiental puede ser un riesgo para la salud laboral del personal dental y alienta a los profesionales a tomar todas las precauciones para minimizar los riesgos asociados.

La presente revisión bibliográfica tiene por objetivo determinar los efectos en la salud y riesgo ocupacional en profesionales de la salud por exposición a óxido nitroso en Odontología.

Metodología: Se realizó una búsqueda en la base de datos Pubmed y Scielo con los términos "Nitrous Oxide" AND "Conscious Sedation" AND "Occupational exposure" AND “Dentistry" obteniendose un total de 29 artículos, de los cuales se incluyeron 11.

Los criterios de inclusión utilizados fueron artículos relacionados con el tema de estudio, idioma de publicación inglés, español o francés, año de publicación desde el año 2010 en adelante y acceso a texto completo.

Resultados y Discusión: Las exposiciones agudas al óxido nitroso causan trastornos, que tratados adecuadamente pueden curarse en un período corto. La exposición crónica induce efectos dañinos en la salud, que ocurren tiempo después de la exposición y pueden durar meses o años. Los más frecuentes son trastornos neurológicos, alteraciones hematológicas y toxicidad del sistema reproductivo.

Conclusión: Los profesionales que realizan sedación con óxido nitroso deben conocer y cumplir con los estándares y prácticas de trabajo adecuadas. La mantención de los equipos junto a una ventilación adecuada es fundamental para la reducción de la exposición y los efectos de salud asociados. Se recomienda monitorear constantemente los niveles de óxido nitroso en el ambiente para garantizar que se cumplan los límites de exposición laboral y evitar efectos dañinos en la salud.

\section{Diferencias en Salud Oral de niños inmigrantes y niños nativos}

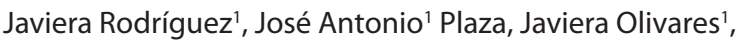
Gonzalo Rodríguez ${ }^{2}$

'Facultad de Odontología, Universidad de Chile. Santiago, Chile. 2Departamento de Odontología Restauradora, Facultad de Odontología, Universidad de Chile.

Introducción: La migración es uno de los fenómenos más antiguos en la historia, que hoy en día cobra una importancia cada vez mayor en la realidad mundial. Además, corresponde a un determinante social de salud, ya que conlleva cambios en los estilos de vida y entorno social de los individuos que la realizan. A pesar de esto, pocos investigadores han estudiado la migración como un determinante social específico en salud oral.

Objetivo: Determinar si existen diferencias en salud oral de niños inmigrantes y niños nativos.

Método: Se realizó una revisión sistemática. Para la búsqueda de información se empleó Pubmed con el algoritmo de búsqueda "Emigrants and Immigrants"[MeSH Terms]" y se le agregó el boleando AND y "Child [MeSH Terms]" AND "Oral Health"[Mesh Terms]. Luego se utilizaron los filtros de estudios realizados en humanos y artículos de máximo 10 años, obteniendo 19 resultados de los cuales se seleccionaron 12 según su pertinencia de título y contenido del abstract.

Resultados: En los artículos revisados se evidencia una relación entre migración y estado de salud oral. Los niños inmigrantes presentan peor salud oral que los niños nativos del país receptor. Además, los inmigrantes recién llegados exhiben peor salud oral que su contraparte no recién llegados, ya que las familias inmi- 
grantes recién llegadas enfrentan barreras idiomáticas, culturales y financieras que limitan el acceso a los seguros de salud y por consiguiente a la atención odontológica.

Conclusión: Se puede concluir de acuerdo a la evidencia que en general los niños inmigrantes tienen peor salud oral que los niños nativos. Por lo que existe la necesidad de implementar programas interculturales de prevención y atención de salud oral dirigidos a niños inmigrantes y sus cuidadores, que se adecuen a su propia realidad y cultural.

\section{Diagnósticos diferenciales de aumentos de volumen en el piso de boca}

Cristina Bofarull', Josefina Bendersky', Luis Romo'.

'Escuela de Odontología, Facultad de Medicina, Pontificia Universidad Católica de Chile. Santiago, Chile

Introducción: El piso de boca contiene diferentes estructuras anatómicas y distintos tipos de tejidos, lo que explica la presencia de diferentes patologías en esta zona, las cuales, al generar aumentos de volumen, teniendo en algunos casos presentación clínica similar, se dificulta el proceso de diagnóstico, pudiendo generar errores en el manejo y planificación de tratamientos.

Objetivo: Describir los diagnósticos diferenciales ante un aumento de volumen en el piso de boca, según la evidencia disponible.

Materiales y métodos: Se realiza una búsqueda sistemática, utilizando las bases de datos Pubmed, Google Scholar y Cochrane Library y Epistemonikos. Se seleccionan 12 estudios utilizando diferentes criterios de inclusión y exclusión.

Resultados: Al presentar un paciente un aumento de volumen en el piso de boca, existen varias hipótesis diagnósticas. Hemos creado un diagrama de manera de facilitar este proceso. Primero, es relevante saber la historia de evolución de la lesión. En el caso de ser rápida, se puede tratar de una ránula o de un absceso de origen dentario. Si la evolución es lenta, podría ser un lipoma, un quiste dermoide, un higroma quístico, un adenoma pleomórfico o un carcinoma mucoepidermoide, entre otros. Luego, se identifican signos clínicos característicos, como color y consistencia, sumado a la presencia o ausencia de dolor. Como tercer paso, se llega al diagnóstico mediante las características de la Resonancia Magnética, confirmado el diagnóstico con la histopatología.

Discusión y conclusión: El diagnóstico diferencial de estas patologías es de suma importancia, confirmándolo siempre con la histopatología pertinente, de manera de realizar el tratamiento oportuno de la lesión y poder pesquisar tempranamente la presencia de un tumor maligno.

\section{Neuralgia por invasión perineural asociada a carcinoma invasor: Revisión bibliográfica a propósito de un caso}

Romina Aguilar ${ }^{1}$, Melissa Pacheco ${ }^{1}$, Macarena Uribe ${ }^{1}$, Natalia Zúñiga', Andrea Cariz'1, Verónica Palacios ${ }^{1}$

'Escuela de Odontología, Facultad de Medicina, Pontificia Universidad Católica de Chile. Santiago, Chile

Introducción: El cáncer oral es uno de los diez cánceres más comunes a nivel mundial. Presenta una detección clínica tardía y por consiguiente un mal pronóstico. Muchas veces los pacientes son diagnosticados tardíamente cuando padecen dolor asociado al carcinoma. Reportes señalan que $86 \%$ de los pacientes con cáncer manifiesta dolor orofacial siendo este uno de los peores síntomas.

Objetivos: Recopilar y describir conceptos actualizados sobre neuralgia en cáncer oral asociado a invasión perineural.

Material y métodos: Se realizó una búsqueda en las bases de datos MEDLINE y Google Scholar. Se aplicaron los criterios de inclusión: artículo publicado entre años 2009 y 2019, escritos en inglés, de acceso gratuito, disponibles en toda su extensión. Se aplicaron los criterios de exclusión: año de publicación anterior a 2009, idioma distinto a inglés, sin términos claves (perineural invasion/oral/cancer AND pain), artículos duplicados. Se seleccionaron 25 artículos para la presente revisión narrativa.

Resultados: El dolor asociado a cáncer oral puede deberse a invasión perineural (PNI). Las células tumorales se diseminan a lo largo del endo o perineuro debido a un entorno privilegiado. Los nervios más comprometidos son el trigémino y facial. Clínicamente destacan dolor, parestesia, anestesia y asimetría facial, aunque un elevado porcentaje de pacientes (35-40\%) puede encontrarse asintomático. La resonancia magnética con contraste se constituye como el examen radiográfico más sensible (95\%) para detectar la diseminación perineural.

Discusión: El mecanismo biológico de la patogénesis de PNI no se conoce bien, existe la teoría de interacciones de señalización recíproca y la capacidad (adquirida) de las células tumorales para responder a las señales dentro del nervio periférico, que promueven invasión. 
Conclusiones: La neuralgia por invasión perineural es un signo de metástasis, mal pronóstico y sobrevivencia $<5$ años. Existen mediadores nociceptivos que sensibilizan los nociceptores aferentes primarios en el microambiente del cáncer.

\section{Living FRISBEE: Fluoruro Diamino de Plata versus Técnica Restauradora Atraumática para el arresto de caries}

\author{
Juan Pablo Vargas ${ }^{1}$, Wenliang Lai ${ }^{1}$, Macarena Uribe ${ }^{1}$, Francisca \\ Verdugo $^{2,3}$, Duniel Ortuño' \\ 'Escuela de Odontología, Facultad de Medicina, Pontificia Universidad \\ Católica de Chile. Santiago, Chile ${ }^{2}$ Centro Evidencia UC, Pontificia Universidad \\ Católica de Chile ${ }^{3}$ Epistemonikos Foundation
}

Las lesiones de caries cavitadas en dentición primaria y mixta requieren un diagnóstico y tratamiento oportuno. El Fluoruro Diamino de Plata (SDF) ha surgido como una alternativa a la Técnica de Restauración Atraumática (ART) debido a su fácil aplicación. Sin embargo, aún existe incertidumbre en relación a su efectividad y seguridad.

Realizamos una búsqueda en Epistemonikos, la mayor base de datos de revisiones sistemáticas (RS) en salud, la cual es mantenida mediante búsquedas en múltiples fuentes de información, incluyendo MEDLINE, EMBASE, Cochrane, entre otras. Extrajimos los datos desde las RS identificadas que comparan SDF versus ART para el arresto de caries en niños. Reanalizamos los datos de los estudios primarios (EP), realizamos un metanálisis y preparamos una tabla de resumen de los resultados utilizando la metodología GRADE. Todos los procesos se realizaron por duplicado y revisados por un equipo metodológico y clínico.

Identificamos 10 revisiones sistemáticas que en conjunto incluían $2 \mathrm{EP}$, ambos ensayos clínicos aleatorizados (ECAA) que incluían niños de entre 3 y 6 años con caries cavitadas sin compromiso pulpar. Ambos ECAA incluyeron 303 pacientes donde la unidad de análisis fue el diente $(n=953)$. El SDF en comparación al ART podría mejorar el arresto de lesiones cariosas en dentición primaria y mixta (baja certeza de la evidencia) RR 1.19 [0.94-1-50]. Por otra parte, no es posible establecer con claridad si el tratamiento con SDF aumenta los efectos adversos debido a que la certeza de la evidencia existente se evaluó como muy baja. Ninguna de las RS evaluó los valores y preferencias de los pacientes.

El SDF ha probado ser efectivo en el arresto de caries, evitando la ejecución de acciones clínicas más invasivas. De todas formas, existe incertidumbre en relación a sus efectos adversos. Se requieren nuevos ECAA que evalúen su efectividad, seguridad y preferencia de los pacientes.

\section{Caries Living Overview of Evidence (L-OVE) vs. Cochrane Library of Systematic Reviews}

\author{
Magdalena Yarur ${ }^{1}$, Vicente Córdova ${ }^{1}$, Martín Chavarría', Juan \\ Pablo Vargas', Wenliang Lai', Duniel Ortuño' \\ 'Escuela de Odontología, Facultad de Medicina, Pontificia Universidad \\ Católica de Chile. Santiago, Chile
}

Las Revisiones Sistemáticas (RS) son consideradas la mejor fuente de evidencia, sin embargo, producirlas requiere tiempo y recursos. En odontología, en los últimos años ha aumentado el número de RS. Reutilizar la información previa, encontrada por otras RS puede ser eficiente. Living Overview of the Evidence (L-OVE) es una plataforma que reúne la evidencia más relevante sobre un tópico biomédico en Epistemonikos. Caries L-OVE contiene toda la información actualizada sobre prevención de caries.

Se incluyeron todas las RS Cochrane Oral Health Group, publicadas hasta marzo de 2019. Solo se incluyeron RS de ensayos clínicos randomizados o quasi randomizados, y se registró el número de Estudios Primarios (EP) de cada RS. Utilizando matrices de evidencia, se determinó cuál de estos EP ya habían sido incluidos en otra RS del mismo tópico.

Se seleccionó un total de 22 RS Cochrane y 406 RS no-Cochrane sobre prevención de caries. De las anteriores, una RS Cochrane no incluyó ningún EP y otras dos incluyeron estudios observacionales que no se consideraron dentro del análisis. Un total de 218 EP (media de RS: 13) fueron utilizados en 19 RS Cochrane para prevención de Caries; 191 de ellas (media de RS: 11) ya habían sido incluidas en RS previas, de modo que, el $84,7 \%$ de los EP incluidos en RS Cochrane ya habían sido incluidas en RS no-Cochrane previamente publicadas. En adición, las RS Cochrane no incluyeron 29 EP (media de RS: 2) que sí fueron incluidos en Caries L-OVE.

Un alto número de EP relevantes de RS Cochrane ya habían sido considerados por otras RS previas. Parece ser más eficiente realizar una búsqueda y selección de artículos previamente incluidos en RS, que conducir una búsqueda desde cero. 


\section{Cuantificación de Neuropilina-1 en fluido gingival crevicular en individuos con diferente severidad de enfermedad periodontal}

Gloria Maurer', Aldo Figari', Diego Prieto'

${ }^{1}$ Facultad de Odontología, Universidad de los Andes. Santiago, Chile.

Introducción: El nuevo sistema de clasificación de enfermedad periodontal propone el uso de biomarcadores como factores a considerar ya que estos pueden contribuir en la detección temprana de periodontitis (1). En el fluido gingival crevicular (FGC) se pueden encontrar distintos mediadores, los cuales se han utilizados para determinar el estado inflamatorio del tejido periodontal (2). Se ha considerado que en éste se podría detectar la presencia de Neuropilina-1 soluble (sNRP-1), que son glicoproteínas de transmembrana que cumplen funciones angiogénicas y reguladoras de la respuesta inmune $(3,4)$; procesos íntimamente relacionados a su vez con la enfermedad periodontal $(5,6)$.

Pregunta de investigación: ¿Existen diferencias en los niveles de sNRP-1, que se obtendrán en FGC de individuos con diferente tipo y severidad de enfermedad periodontal según la nueva clasificación del año 2017, en pacientes adultos atendidos en el Centro de Salud de San Bernardo (CESA) de la Universidad de los Andes durante el periodo académico 2019-2020?.

Metodología: Se realizará un estudio de corte transversal en el cual se reclutarán 135 participantes voluntarios que asistan a la unidad de diagnóstico del CESA en el período académico 2019-2020. La toma de FGC será efectuada por dos evaluadores calibrados y se realizará mediante PerioPaper Strips ${ }^{\circledR}$ con aislación relativa, las que se almacenarán en tubos Eppendorf $\mathrm{a}-80^{\circ} \mathrm{C}$. Las muestras se procesarán mediante Test ELISA en el centro de investigación biomédica de la Universidad de los Andes. El análisis estadístico de los datos obtenidos se realizará mediante el uso del software STATA 14.2.

Resultados esperados: Se espera que sNRP-1 se encuentre en asociación con periodontitis, y a su vez, aumente sus concentraciones de manera proporcional al tipo y severidad de enfermedad periodontal.

\section{Conocimiento sobre salud bucal de educadores de párvulos intervenidos y no intervenidos por sembrando sonrisas}

\author{
Carla Madrid', Dusan Marinkovic', Jessica Matute', Valentina \\ Nuñez ${ }^{1}$, Josefina Opazo' ${ }^{1}$, Cynthia Cantarutti ${ }^{1}$ \\ 'Escuela de Odontología, Facultad de Medicina, Pontificia Universidad \\ Católica de Chile. Santiago, Chile.
}

Introducción: Los educadores representan una autoridad influyente en el conocimiento y en los hábitos de los preescolares. Actualmente, se han implementado programas para promover la salud bucal y prevenir enfermedades, como el programa Sembrando Sonrisas (PSS). Sin embargo, a pesar de que existe evidencia disponible que evalúa la eficacia de estos programas, no existen estudios comparativos que demuestren qué saben, cuánto saben y qué aplican en sus prácticas los educadores de párvulos que reciben el PSS.

Objetivos: Comparar el conocimiento y aplicación de medidas preventivas de caries y gingivitis en preescolares, de los educadores de párvulos de jardines infantiles intervenidos por el PSS en comparación a un grupo no intervenido, en la comuna de El Bosque.

Metodología: Se realizará un estudio de diseño tipo mixto, transversal para la metodología cuantitativa y fenomenológico para el estudio cualitativo. La población en estudio serán los educadores de jardines infantiles intervenidos por el PSS de la comuna de El Bosque y educadores de jardines no intervenidos. Para el subestudio cuantitativo, la muestra corresponderá a la población total. Para el subestudio cualitativo, la muestra será estimada mediante un muestreo por conveniencia por conglomerados. Para la recolección de datos se utilizarán entrevistas semiestructuradas con preguntas norteadoras. Para el análisis de los resultados del subestudio cuantitativo se utilizará programa Excel y SPSS Statistics V25. El análisis del subestudio cualitativo se hará mediante lectura de las narrativas, levantando unidades de análisis según temas emergentes. La confiabilidad será entregada según la pauta CASPE, triangulación en entrevista, análisis e incorporación de información de un estudio mixto y member check.

Resultados esperados: Los educadores de jardines intervenidos por el PSS conocen y aplican más estrategias de prevención de enfermedades y promoción de hábitos de higiene oral, en comparación con los educadores de jardines que no son intervenidos por el programa. 